Thorax (1973), 28, 222.

\title{
Scanning electron microscopy of pulmonary alveolar capillary vessels
}

\author{
I. G. S. ALEXANDER ${ }^{1}$, B. C. RITCHIE, and J. E. MALONEY \\ Departments of Anatomy and Medicine, Monash University, and Department of Physiology, \\ Melbourne University, Victoria, Australia
}

\begin{abstract}
The pattern of subepithelial vessels in pulmonary alveoli of rabbits has been studied using scanning electron microscopy.

Alveolar capillaries form a network of interconnecting vascular rings, most of which surround the periphery of type II cells of the alveolar epithelium. Individual capillaries contributing to the formation of adjacent rings follow a corrugated course with angulations located on the sites of junction with other capillaries completing the rings; the capillaries are covered by type I epithelial cells which also extend into and form the alveolar lining at the peripheral area of the interstices of the capillary network. Single type II cells form the alveolar lining at the centre of vascular rings.

The pattern of pulmonary alveolar capillaries revealed by scanning electron microscopy is thus similar to that postulated by Weibel (1963) on the basis of transmission microscopic studies.
\end{abstract}

Scanning electron microscopy has been used extensively for some years, but it is only recently that suitable techniques for the study of biological specimens have become available. The suggestion of Barber and Boyde (1968) that specimens should be embedded in plastic and the excess material eroded by solvents, was taken up by Cleveland and Schneider (1969) and further modified by ourselves (Alexander, Ritchie, and Maloney, 1971). The development of these techniques has made it possible to re-examine the mammalian pulmonary alveolar surfaces which are particularly suitable for study with the scanning electron microscope by utilizing the large depth of focus of this instrument.

Weibel and Gomez (1962) and Weibel (1963) studied pulmonary alveolar capillaries using transmission microscopy and concluded that the most probable arrangement of alveolar capillaries was in the form of a hexagonal network. The results of the present study largely confirm their observations.

\section{MATERIALS AND METHODS}

Rabbits were killed by an overdose of sodium pentobarbitone administered intravenously. The trachea was tied off by double ligatures and the chest was

1Correspondence to: Dr. I. G. S. Alexander, Department of Anatomy, Monash University, Clayton, 3168, Victoria, Australia opened. A ligature was placed around the major $\stackrel{\nexists}{\circ}$ vessels at the base of the heart after which the heart $\stackrel{\Phi}{\Omega}$ and lungs were excised in toto. The intratracheal pres- $\overrightarrow{\vec{O}}$ sure at this stage was $1 \mathrm{~cm} \mathrm{H}_{2} \mathrm{O}$.

The lungs were immersed in $10 \%$ neutral formalin at $4^{\circ} \mathrm{C}$ for two days. Subsequently the upper azygos lobe of the lungs was separated and cut into slices approximately $1 \mathrm{~mm}$ thick. The slices were then immersed in $5 \%$ phosphate-buffered glutaraldehyde, pH 7.4 (Sjöstrand, 1967) for 4 hours at $4^{\circ} \mathrm{C}$. $\times$ The tissue slices were washed in the phosphate buffer, immersed in $2 \%$ osmium tetroxide at $4^{\circ} \mathrm{C}$. for 1 hour, and dehydrated in graded alcohol $\delta$ solutions.

The pieces of lung were then transferred into an 0 epoxypropane-Araldite mixture and finally embedded in Araldite (Glauert and Glauert, 1958). After 2 hours $\frac{7}{0}$ at room temperature they were polymerized for $30 \cong$. minutes at $60^{\circ} \mathrm{C}$, soaked in acetone for 10 minutes, $N$ and scoured with acetone until all surfaces presented a mat appearance. They were then mounted on metal $\tilde{}$ stubs with a plasticized nitrocellulose solution ${ }^{2}$. The ${ }_{\omega}^{N}$ stubs bearing the specimens were positioned at ano angle of $45^{\circ}$ to an evaporation source and rotated in vacuo while a conducting layer of gold was deposited $\mathrm{C}_{0}$ on all exposed surfaces. The thickness of this layeron was controlled by matching the colour of a similarly exposed tile to a standard. Transmission electron ${ }^{\circ}$ microscopic examination of similarly treated $\stackrel{\odot}{\stackrel{P}{*}}$ material, embedded and sectioned appropriately, $\stackrel{\mathbb{P}}{\mathbb{Q}}$ showed the thickness of the gold layer to be $20 \mathrm{~nm}$. $\stackrel{\mathbb{Q}}{\circ}$

2Colorless Cutex Spillpruf Polish, Chesbrough-Ponds Internationa Lid., Clayton, Victoria 


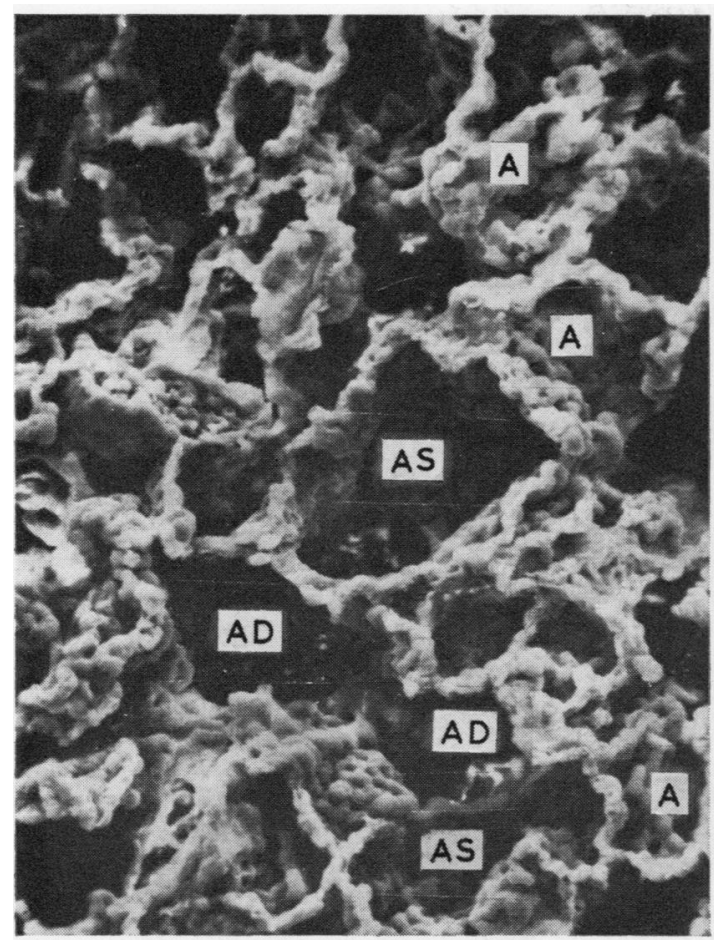

FIG. 1

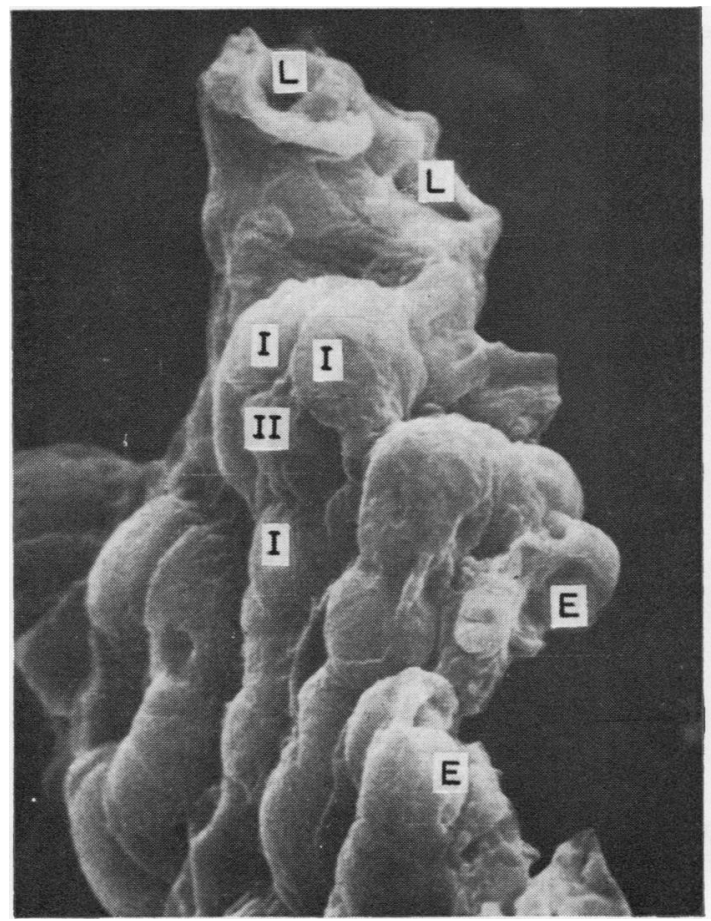

FIG. 3

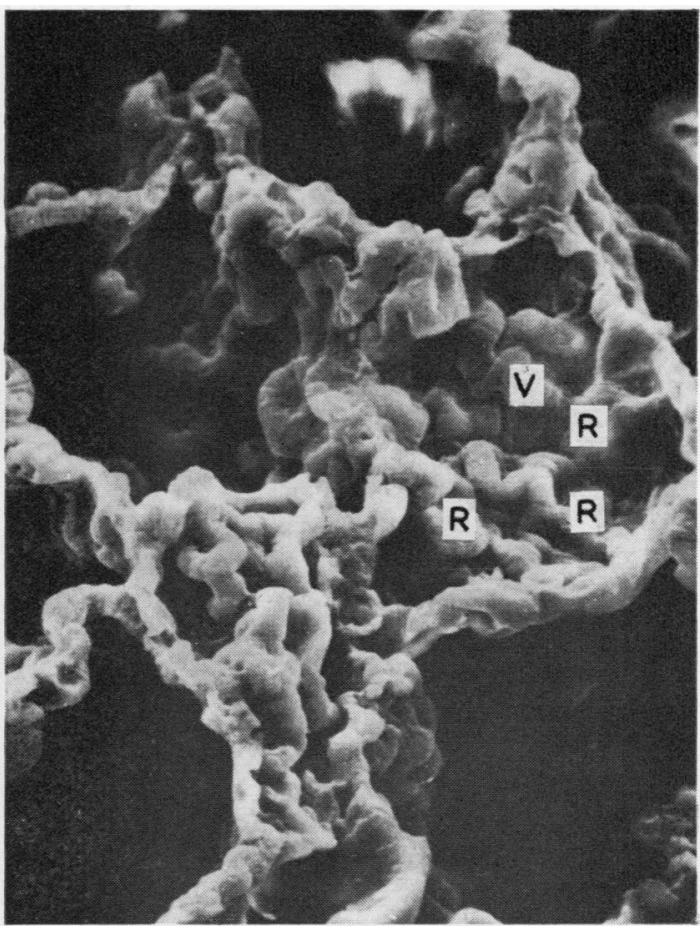

FIG. 2

FIG. 1. Scanning electron micrograph of the cut surface of rabbit lung. Alveoli $(A)$, alveolar sacs $(A S)$, and alveolar ducts $(A D)$ are shown (approximately $120 \times$ ).

FIG. 2. Scanning electron micrograph of rabbit alveoli. Ridges representing subepithelial vessels $(V)$ form interlocking rings $(R)$ (approximately $650 \times$ ).

FIG. 3. Scanning electron micrograph of portion of alveolar wall of rabbit. Lumina $(L)$ of severed vessels are shown. Occasional red cells (E) emerge from the cut capillaries. A number of flat type I cells (I) surround a type II cell (II) (approximately 3,500×). 


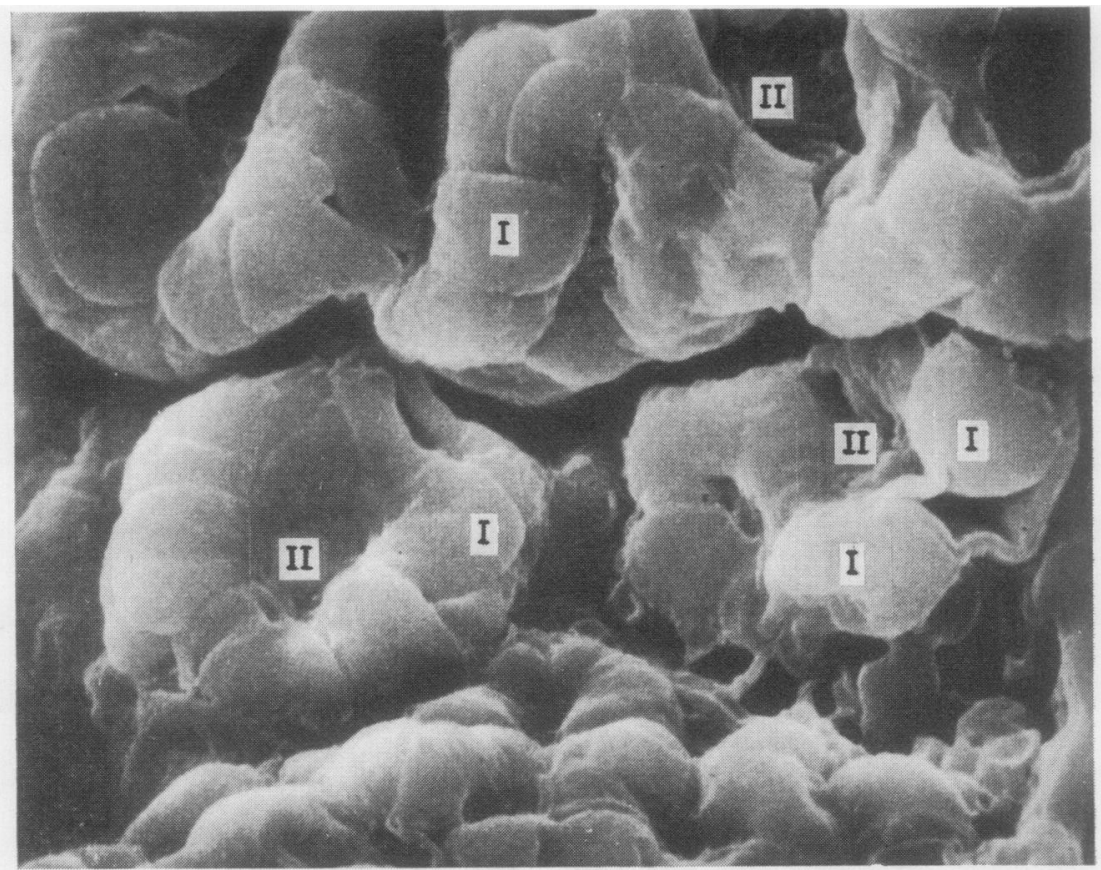

FIG. 4. Scanning electron micrograph of a portion of the pulmonary alveolar surface of rabbit. The rough-surfaced type II cells (II) are in the middle of the vascular rings. The smooth type I cells (I) cover the rings themselves (approximately 2,700×).

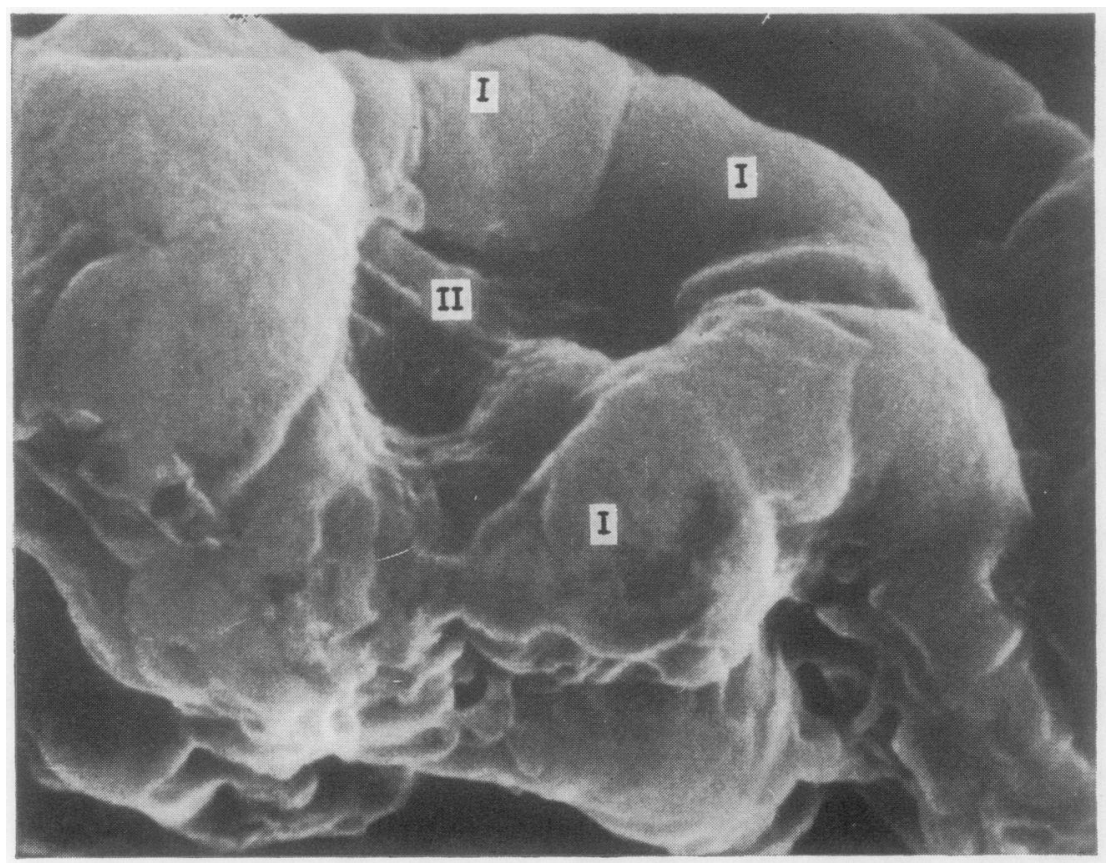

FIG. 5. Scanning electron micrograph of a vascular ring of the pulmonary alveolus of rabbit. A type II cell (II) occupies the centre of the ring; smooth type I cells (I) surround it (approximately 5,500 $\times$ ). 


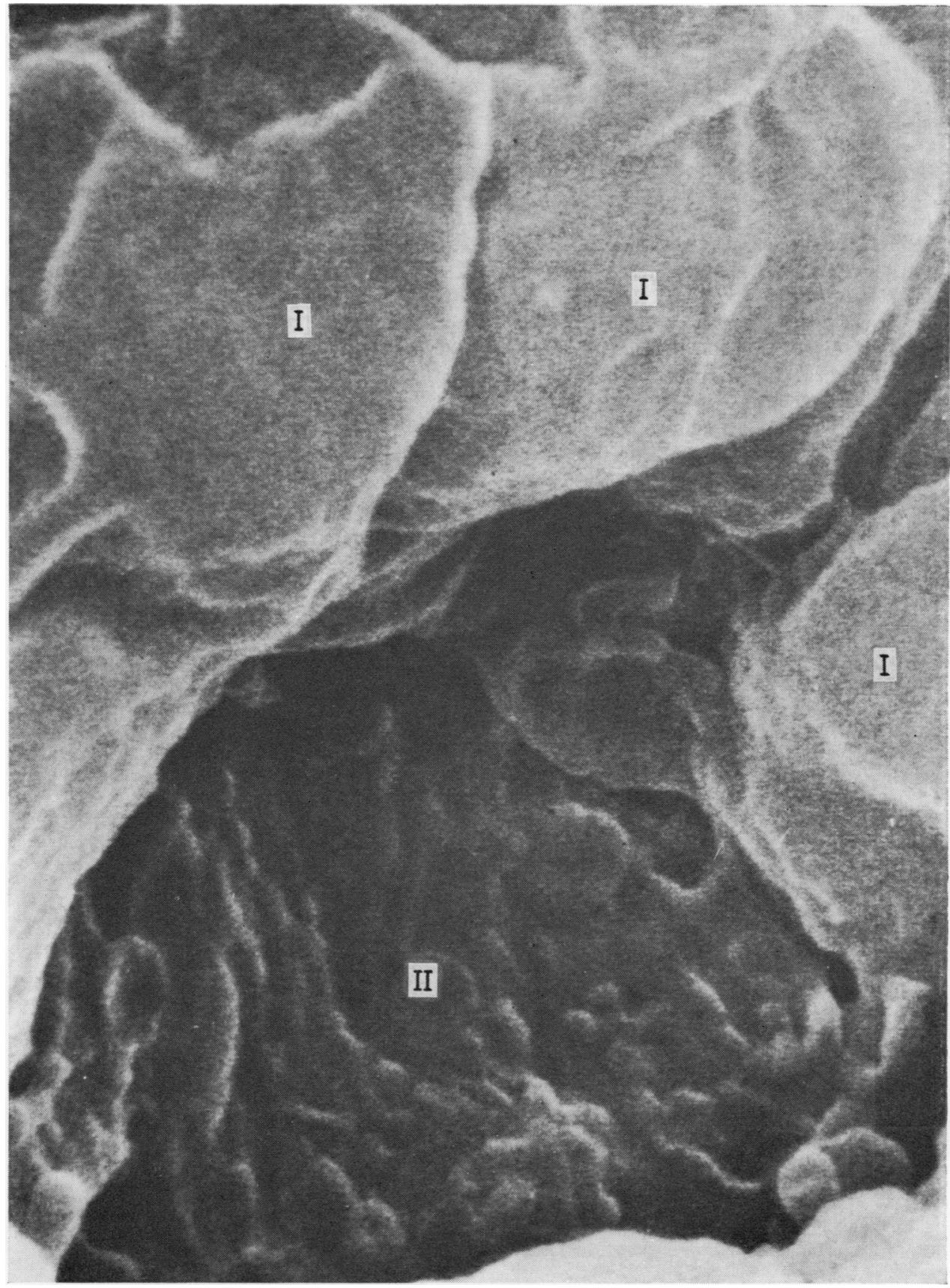

FIG. 6. Scanning electron micrograph of the centre of a vascular ring of rabbit alveolus. A number of smooth type I cells (I) surround a microvillous type II cell (II) (approximately 17,800 $\times$ ). 


\section{RESULTS}

Alveoli, alveolar sacs, and capillary blood vessels can be clearly identified at the exposed surfaces of lungs when examined by scanning electron microscopy (Fig. 1). The alveolar sacs form large spaces and exhibit distinct openings leading to the smaller spaces constituting the alveoli. Cut ends of capillary vessels can be identified and the location of capillaries elsewhere in the alveolar walls can be inferred by continuity between their cut ends and the elevations in the alveolar wall.

The walls of alveoli which have been exposed show a pattern of raised and rounded elevations which form interconnecting rings (Fig. 2). The ridges are formed by subepithelial vessels which occupy the greater part of the alveolar wall and constitute the basis for the pattern of interlocking rings. In some views, erythrocytes can be visualized at the severed ends of vessels in the alveolar wall (Fig. 3). Some of the vessels forming the interlocking rings extend to adjacent alveoli.
Most of the vascular rings seen are approximately $30 \mu \mathrm{m}$ in diameter and the planes of adjacent rings form acute angles with each other (Fig. 5). The vessels connecting adjacent rings and forming part of each ring describe a regular sinuous path with angulations located at the sites of junction with other capillaries. Each vascular ring is covered by 10 to 15 relatively smooth type I epithelial cells separated by intercellular gutters. The type I cells covering the capillaries also form the alveolar lining at the peripheral part of the interstices of the capillary network. The largest transverse dimension of those type I cells in which the whole surface area could be distinguished in one field of view was approximately $20 \mu \mathrm{m}$; the diameter of the underlying capillaries is about $7 \mu \mathrm{m}$. The centres of each ring are occupied by the rougher surfaced type II cell, which is studded by numerous microvilli, 0.25 to $0.5 \mu \mathrm{m}$ in diameter, the total diameter of the cell surface being approximately $9 \mu \mathrm{m}$ (Fig. 6).

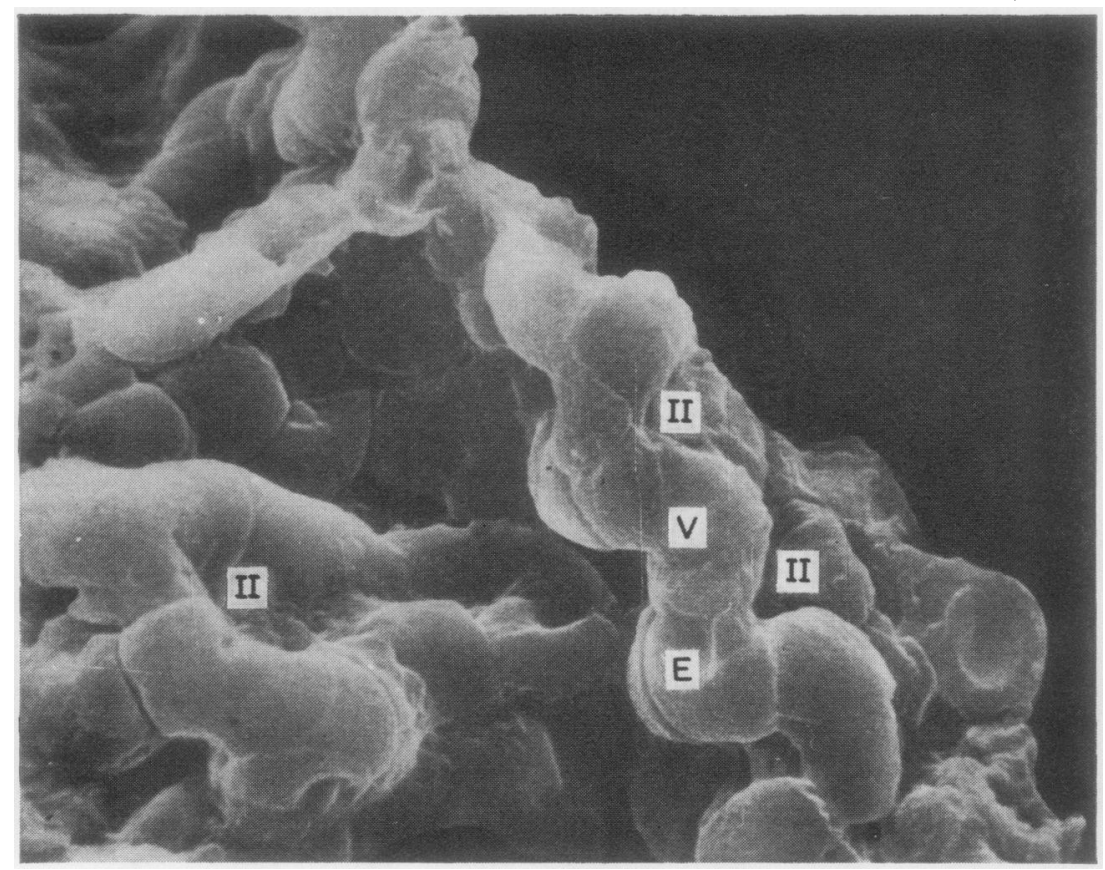

FIG. 7. Scanning electron micrograph of a portion of rabbit pulmonary alveolus. A single vessel $(V)$ forms part of a number of vascular rings surrounding type II cells $(I I)$. The characteristic shape of a red cell $(E)$ appears under the alveolar capillary membrane (approximately $2,100 \times$ ). 


\section{DISCUSSION}

The results of this study using scanning electron microscopy provide direct evidence of a highly organized vascular pattern in the rabbit pulmonary alveolus. Rabbit alveoli show projections which are interpreted as being due to subjacent alveolar capillaries on the basis of their dimensions, the resemblance of their pattern to previously proposed alveolar vascular patterns, and the fact that blood cells are seen emerging from the severed surfaces of underlying capillaries.

Further evidence of the presence of a meshwork of vessels underlying elevations of the alveolar epithelium of the lung comes from the elegant study on mice, hamsters, and rats by Kuhn and Finke (1972).

The system of interconnected vascular rings found in the rabbit by scanning electron microscopy closely resembles the theoretically derived hexagonal meshwork based upon transmission microscopic studies by Weibel (1963). He also predicted that the vessels forming these vascular rings should show sinuous corrugations, an hypothesis confirmed by the results of the present study. The observation that capillaries continue between adjacent alveoli confirms the findings of Staub and Schultz (1968).

The central position of the type II cells in the vascular rings was depicted in passing by Weibel (1963) and their appearance suggests that they act as a cytoarchitectural colonnade around which the vascular structures are gathered. The individual columns of this colonnade, the type II cells, are similar in shape and position to the theoretical posts proposed by Fung and Sobin (1969) which are interposed in the path of blood flow.

The diameters of alveolar surface cells have been derived by Meyrick and Reid (1970) and found to be $54 \mu \mathrm{m}$ in type I cells and $9 \mu \mathrm{m}$ in type II cells. While the latter figure is in excellent agreement with the one found in this investigation, the one quoted for the smooth type I epithelial cells, albeit theoretically derived, is approximately two to four times larger than the one suggested by direct scanning electron microscopic views. These discrepancies may be due to species differences and differing degrees of pulmonary inflation used in the respective studies.

Scanning electron microscopy provides a means of direct examination of the rabbit pulmonary alveolar surfaces. The results of this survey indicate that, except for minor modifications, capillary vessels in pulmonary alveoli form the pattern which was predicted on stereological grounds by Weibel (1963).

This work was supported by grants from the Life Insurance Medical Research Fund of Australia and New Zealand and the Australian Tobacco Research Foundation. The encouragement and advice received from Professor G. C. Schofield is gratefully acknowledged. We should like to thank Dr. D. M. de Kretser for reading the manuscript. The technical assistance of Mrs. Pamela Capicchiano and Miss Marion Exelby is also acknowledged.

\section{REFERENCES}

Alexander, I. G. S., Ritchie, B. C., and Maloney, J. E. (1971). A method of pulmonary tissue preparation for scanning electron microscopy. J. Anat. (Lond.), 108, 215.

Barber, V. C., and Boyde, A. (1968). Scanning electron microscopic studies of cilia. Z. Zellforsch., 84, 269.

Cleveland, P. H., and Schneider, C. W. (1969). A simple method of preserving ocular tissue for scanning electron microscopy. Vision Res., 9, 1401.

Fung, Y. C., and Sobin, S. S. (1969). Theory of sheet flow in lung alveoli. J. appl. Physiol., 26, 472.

Glauert, A. M., and Glauert, R. H. (1958). Araldite as an embedding medium for electron microscopy. J. biophys. biochem. Cytol., 4, 191.

Kuhn, C., and Finke, E. H. (1972). The topography of the pulmonary alveolus: Scanning electron microscopy using different fixations. J. Ultrastruct. Res., 38, 161.

Meyrick, B., and Reid, L. (1970). The alveolar wall. Brit. J. Dis. Chest, 64, 121.

Sjöstrand, F. S. (1967). Electron Microscopy of Cells and Tissues, vol. 1. Academic Press, New York and London.

Staub, N. C., and Schultz, E. L. (1968). Pulmonary capillary length in dog, cat and rabbit. Resp. Physiol., 5, 371.

Weibel, E. R. (1963). Morphometry of the Human Lung. Springer, Berlin. , and Gomez, D. M. (1962). Architecture of the human lung. Science, 137, 577. 